\title{
Optimal Operation of High-Speed Trains Using Hybrid Model Predictive Control
}

\author{
Yingze Yang, Zheng Xu $\mathbb{D}^{\mathbb{D}}$, Weirong Liu, Heng Li $\mathbb{D}^{\mathbb{C}}$, Rui Zhang, and Zhiwu Huang $\mathbb{D}$ \\ School of Information Science and Engineering, Central South University, Changsha 410083, China \\ Correspondence should be addressed to Zhiwu Huang; hzw@csu.edu.cn
}

Received 1 August 2017; Revised 8 January 2018; Accepted 11 April 2018; Published 15 May 2018

Academic Editor: Paola Pellegrini

Copyright (C) 2018 Yingze Yang et al. This is an open access article distributed under the Creative Commons Attribution License, which permits unrestricted use, distribution, and reproduction in any medium, provided the original work is properly cited.

\begin{abstract}
The high-speed train operation process is highly nonlinear and has multiple constraints and objectives, which lead to a requirement for the automatic train operation (ATO) system. In this paper, a hybrid model predictive control (MPC) framework is proposed for the controller design of the ATO system. Firstly, a piecewise linear system with state and input constraints is constructed through piecewise linearization of the high-speed train's nonlinear dynamics. Secondly, the piecewise linear system is transformed into a mixed logical dynamical (MLD) system by introducing the auxiliary binary variables. For the transformed MLD system, a hybrid MPC controller is designed to realize the precise control under hard constraints. To reduce the online computation complexity, the explicit control law is computed offline by employing the mixed-integer linear programming (MILP) technique. Simulation results validate the effectiveness of the proposed method.
\end{abstract}

\section{Introduction}

With rapid development of high-speed railway, the operation safety, punctuality, and energy consumption of train have received more and more attentions. Nowadays, in China, the high-speed railway generally adopts distributed traction power to improve speed and traction efficiency, where locomotives and wagons are marshalled together to form a high-speed train [1]. For distributed traction power trains, there are some critical issues that need to be solved, such as vehicle traction and brake force allocation, operation safety, punctuality, and energy consumption [2].

To achieve the safety and multiobjective optimization, automatic train control system is developed for high-speed railway, and it is one of the key on-board equipment with high safety assurance [3]. The automatic train operation (ATO) system is the essential component that plays a key role in the train operation process [4]. The ATO system controls the train traction/brake force to follow the reference speed. Moreover, ATO system can reduce the energy consumption and improve riding comfort. Now, it is still a challenge to develop an efficient modeling and control method for ATO system [5].

Currently, the researches of automatic train control are mainly based on single-point model and multipoint model [6]. For single-point model, the whole high-speed train is simplified as a single rigid mass point, which ignores the coupling characteristics among the vehicles and the allocation of the distributed traction/brake force. The dynamic properties of single-point model are simple and easy to design. However, high-speed train adopts distributed traction power mode; thus the single-point model is obviously not able to describe the coupling characteristics and hydrodynamic dispersion characteristics of the train. Thus multipoint model is favoured to study the automatic train control strategy.

Yang and Sun $[7,8]$ analyzed the coupling characteristics among vehicles of high-speed train and established train multipoint dynamical model. For both push-pull driving and distributed driving train types, the hybrid $\mathrm{H}_{2} / \mathrm{H}_{\infty}$ automatic train controller was designed and was compared by $\mathrm{H}_{2}$ controller and $H_{\infty}$ controller, respectively. Lin et al. [9] proposed a control strategy based on improved sliding mode control to overcome the effect of the disturbance in the process of train operation, which had good robustness and effectively restrained the high frequency chattering phenomenon. Thus the work reduced the impact of the frequent switching of control input and increased riding comfort. To simplify the controller design, Song et al. [10] utilized the geometric 
topology to reduce multiple positions to one position for multipoint model. In their work, to address the nonlinear saturation constraints of traction and brake forces, the robust adaptive controller was designed with low computing load, which had robustness for train disturbance and uncertainty of model parameters.

Based on the multipoint model, Zhuan and Xia [11] have achieved constructive results on the automatic train control strategy of the heavy-duty train in South Africa. Specifically, Zhuan and Xia [12] put forward three kinds of offline open-loop optimal operation strategies, whose goals are to minimize the workshop bonding force, ensure the safety of train driving, and reduce the maintenance costs of the coupler buffer. Then, Chou and Xia [13] proposed a closed-loop cruising linear quadratic regulator controller to minimize the running cost of electric air brake system in heavy-haul train, in which the control objective includes the speed tracking, coupling force, and energy consumption. In order to overcome the communication limitation, the vehicle barrier was introduced and the controller was reconstructed based on the current orbital slope. In addition, Zhuan and Xia [14] adopted the output feedback adjustment method to regulate the speed of the heavy train and verified the application conditions of their proposed method.

As a summary, Scheepmaker et al. [15] presented an extensive review on energy-efficient train control and the related topic of energy-efficient train timetabling, from the first simplest model of a train running on a level track to the advanced models and algorithms of the last decade dealing with varying gradients and speed limits and including regenerative braking. And there have appeared various theories and technologies to realize the efficient and safety train operation, such as the passivity-based cruise control [16], the robust adaptive control [17], and the iterative learning and fault detection approaches $[18,19]$.

$\mathrm{Li}$ et al. [20] considered the optimal guaranteed cost of cruise control for high-speed train movement. The sufficient condition for the existence of guaranteed cost cruise control law is given in terms of linear matrix inequalities. And a convex optimization problem is formulated to determine the optimal guaranteed cost that minimizes the performance upper bound of the cruise control law. Ye and Liu [21] propose a novel approach to solve the complex optimal train control problems in closed loop by introducing some simplifications. The operation sequence consists of maximum traction, speed holding, coasting, and maximum braking on each subsection of the track or a constant force is applied on each subsection. Zhao et al. [22] proposed a new cluster consensus technique to design the distributed control law, by which the trains can track the desired speeds asymptotically and the distance between the neighboring cars can be kept in the ideal range.

However, with the improvement of the requirement for ATO control performance, the above methods lack the ability to address the nonlinear, multiconstraint, and multiobjective problem based on the multipoint model, which leads to their limitations in practical applications. Especially for online operation process of high-speed trains, it raises the nonlinear resistance force which makes many classical control methods based on the linear resistance force model or the single equilibrium point linearizable model difficult to be implemented. And it is necessary to consider the safe speed, the saturation characteristics of traction and braking units, and the work conditions such as maximization of bonding forces, operating punctuality, energy-efficiency, and reduction of coupling wear forces. The model predictive control has the advantages of fully considering the input and state constraints of the system and the multiobjective optimization problems in the design of the controller, which is more suitable for the design of ATO controller [23, 24].

In this paper, an optimal automatic train control strategy is proposed for high-speed trains based on model predictive control. Due to the high computation complexity of nonlinear train model, the common method is to linearize the nonlinear system model at the equilibrium points [25]. However, if the nonlinear system has a wide range of operating condition, there are many deviations on entire running process for singular linearized model with one equilibrium point. Thus, the piecewise linear systems are utilized to approximate the original system, which can describe complex nonlinear systems accurately [26]. In this paper, the nonlinear running resistance is fitted by multiple line segments to construct the piecewise linear system model of train.

In the traditional controller design based on piecewise linear model, the linear submodel will be determined according to the initial state of receding horizon; then the predicted control is solved based on the determined sublinear model, which is fixed in the entire receding horizon [27]. However, for each actual control step, the system state changes and the corresponding submodel switch may also occur. The fixed submodel is difficult to approximate the original nonlinear model, especially near the model switching point. Therefore, the traditional model prediction control just according to current linear submodel can result in a large prediction error.

In order to solve the above problems, the logic variables are introduced to describe the switch of different linear submodels in this paper. By using this method, the piecewise linear system can be transformed into a hybrid logic dynamical system [28, 29]. For the hybrid logic dynamical system, the model switch can be described by changing the logical variables. Then the hybrid model predictive control (MPC) can be utilized to generate the control inputs based on the constructed mixed logic dynamical system.

The hybrid MPC controller uses the mixed logic dynamical model to predict the future evolution of the system at each time step, and a certain performance index is optimized under operating constraints with respect to a sequence of future input moves. The first of such optimal solution is the control action applied to the plant. For the hybrid MPC controller, the optimal problem that needs to be solved is a mixed integer linear program (MILP) due to the hybrid system description. Because the mixed logic dynamical model ensures that the submodel can switch on the entire receding horizon. Thus, more accurate approximation can be obtained by hybrid MPC and the prediction error is reduced.

Compared to the existing work, the proposed hybrid MPC makes a superior trade-off between the model complexity and control performance. The proposed controller can obtain the optimal input sequence without excessive 


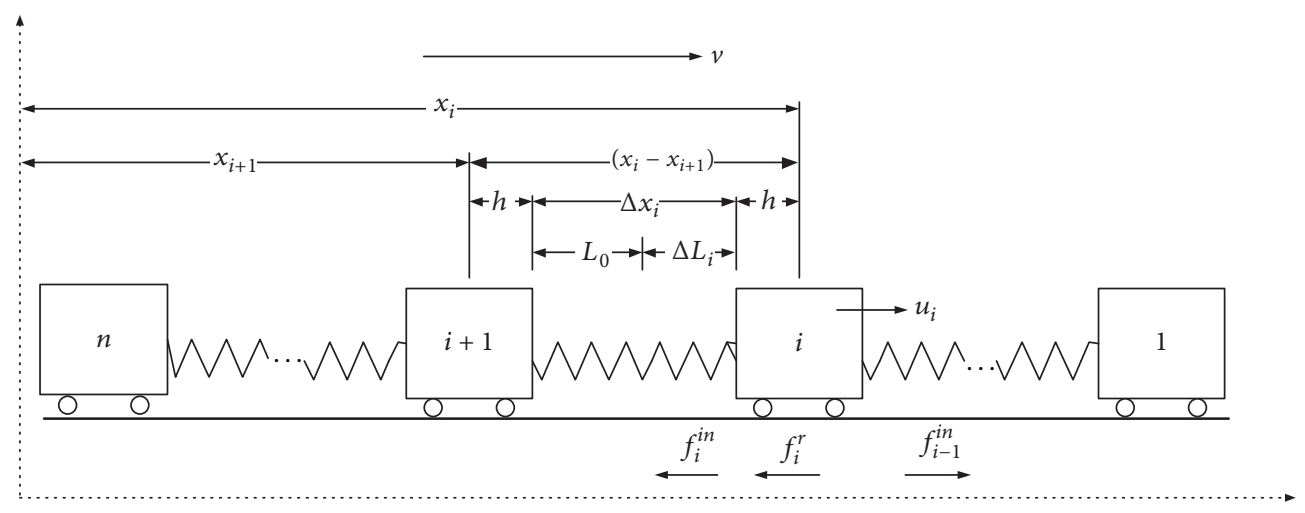

FIgURE 1: The multiple mass-point model structure of the electric multiple units.

simplifying assumption, while the piecewise linear model reduces the computation complexity under the sufficient precision compared to the classical MPC method. The main contributions in this paper can be summarized as follows.

(1) The nonlinear multiple mass point dynamical model of high-speed trains is constructed. In the model, the complex nonlinear characteristics of running resistance are piecewise linearized and the piecewise linear description is transformed to mixed logic dynamical system.

(2) Based on the MLD system model, a hybrid MPC framework is introduced and the MLD model is used as the prediction model. Based on the MLD prediction model, the control problem can be formulated as a unified mixed integer linear programming (MILP) problem, even the piecewise linear characteristics of train model.

(3) For the formulated MILP problem containing logical variables and continuous variables, the branch and bound method $(B \& B)$ is utilized to transform the original MILP problem into linear programming (LP) by relaxing the logic variable constraints.

The remainder of the paper is organized as follows. In Section 2, we establish the piecewise linear model of the high-speed train and transform the piecewise linear model to MLD model. In Section 3, a mixed logic dynamical system is developed. In Section 4, a hybrid MPC controller is designed by analyzing the performance indices and constraints of highspeed trains. Then, the simulation results are provided to validate the effectiveness of the controller in Section 5. Finally, the major conclusion of this paper is given in Section 6 .

\section{The Model of High-Speed Train}

In this section, we establish the nonlinear multiple masspoint dynamic model of high-speed trains by analyzing their dynamical characteristics. Because the nonlinear model is complex for designing the system controller, the nonlinear curve of the running resistance is piecewise linearized and the piecewise linear model of the train is constructed.

2.1. The Multiple Mass-Point Model of Trains. Figure 1 presents the multiple mass-point model structure for the simplified electric multiple power units. The total number of locomotives and wagons is denoted by $n$. These vehicles are connected by the vehicle hook couplers. The mechanical properties of the couplers are usually described as the "elasticdamping" system. Thus, the hook coupler force between vehicle $i$ and vehicle $i+1$ is as follows:

$$
f_{i}^{i n}=k_{i} \Delta L_{i}+d_{i} \Delta \dot{L}_{i},
$$

where $\Delta L_{i}=x_{i}-x_{i+1}-h_{i+1}-h_{i}-L_{0}$ is the offset value of the relative equilibrium position of the hook coupler between vehicle $i$ and vehicle $i+1, L_{0}$ is the natural length of the coupler and $h_{i}$ is the half length of vehicle $i$, and $x_{i}$ is the displacement of vehicle $i$. Since the length of each vehicle is essentially the same and constant (denoted by $h$ ) and the hook coupler has the same type, the balance position between two vehicles can be denoted by $L_{e}=2 h+L_{0}$. Thus $\Delta L_{i}=x_{i}-x_{i+1}-L_{e}$. And the hook coupler force (1) can be transferred to

$$
f_{i}^{i n}=k\left(x_{i}-x_{i+1}-L_{e}\right)+d\left(\dot{x}_{i}-\dot{x}_{i+1}\right) .
$$

The multiple mass-point dynamic equation of electric multiple power units can be described as

$$
\begin{aligned}
m_{1} \dot{v}_{1} & =u_{1}-f_{1}^{i n}-f_{1}^{r}, \\
m_{i} \dot{v}_{i} & =u_{i}+f_{i-1}^{\text {in }}-f_{i}^{i n}-f_{i}^{r}, \quad(i=2,3, \ldots, n-1) \\
m_{n} \dot{v}_{n} & =u_{n}+f_{n-1}^{\text {in }}-f_{n}^{r}, \\
\dot{x}_{i} & =v_{i}, \quad(i=1,2, \ldots, n),
\end{aligned}
$$

where $u_{i}$ is the control input of vehicle $i$. When the train is in traction state or cruise state, $u_{i}>0$ is traction force. When the train is in brake state, $u_{i}<0$ is the brake force. When the train is in coasting state, $u_{i}=0$, implying that there is no traction force and brake force. For locomotive vehicles, they can provide both traction force and brake force, while for wagon vehicles, they can just provide brake force. Assume that $f_{i}^{r}$ denotes the running resistance of vehicle $i$; that is,

$$
\begin{aligned}
f_{i}^{r}= & f_{i}^{b}+f_{i}^{a} \\
= & m_{i} \mathcal{c}_{0}+m_{i} c_{v} v_{i}+m_{i} c_{a} v_{i}^{2}+m_{i} g \cdot \sin \theta_{i}+0.004 \\
& \cdot m_{i} D_{i},
\end{aligned}
$$

where $f_{i}^{b}$ and $f_{i}^{a}$ are the basic resistance and appended resistance, respectively, and $m_{i}$ and $v_{i}$ are the mass and 
TABLE 1: The basic resistance parameters of Chinese electric multiple units high-speed train CRH3.

\begin{tabular}{lcc}
\hline Parameter & Value & Unit \\
\hline$m_{i}$ & 47.5 & $\mathrm{t}$ \\
$c_{0}$ & $7.75 \times 10^{-3}$ & $\mathrm{Nkg}^{-1}$ \\
$c_{v}$ & $2.28 \times 10^{-4}$ & $\mathrm{Ns}^{-1} \mathrm{~kg}^{-1}$ \\
$c_{a}$ & $1.66 \times 10^{-5}$ & $\mathrm{Ns}^{2}\left(\mathrm{~m}^{2} \mathrm{~kg}^{-1}\right.$ \\
\hline
\end{tabular}

TABLE 2: The piecewise linear parameters of basic resistance.

\begin{tabular}{rrrr}
\hline$j$ & $\alpha_{j}\left(\mathrm{~s}^{-1}\right)$ & $\beta_{j}\left(\mathrm{Nkg}^{-1}\right)$ & Speed $\left(\mathrm{m} \cdot \mathrm{s}^{-1}\right)$ \\
\hline 1 & 0.0008 & 0.0044 & {$[0,35]$} \\
2 & 0.0020 & -0.0363 & {$[35,70]$} \\
3 & 0.0031 & -0.1100 & {$[70,100]$} \\
\hline
\end{tabular}

velocity of vehicle $i, \theta_{i}$ is the ramp angle, and $D_{i}$ is the curvature of rail.

\subsection{The Multiple Mass-Point Piecewise Linearized Model of} Trains. According to the dynamic analysis of high-speed trains, the basic resistance of the train is composed of mechanical resistance and air resistance. When the train is running at low speed, for instance, below $44.44 \mathrm{~m} / \mathrm{s}$ $(160 \mathrm{~km} / \mathrm{h})$, the basic resistance is mainly mechanical resistance, and air resistance can be ignored. However, when the train runs at high speed, air resistance will be dominant, and it is related to the square item of the train speed with nonlinear characteristics. As the speed of high-speed trains becomes higher, this nonlinear relationship is becoming stronger. In order to facilitate the controller design of nonlinear model, we consider linearization of the nonlinear model of the train.

For nonlinear systems, the linear model is usually obtained by using the equilibrium linearization method. However, for high-speed trains with varying speeds, it is not appropriate to choose one equilibrium point. Piecewise linear systems approximate the original system by using multiple linear subsystems, which can approximate most complex nonlinear systems accurately. Thus, the least square method is adopted to fit the train running resistance linearly. By using the method, the piecewise linear system model of the train is established.

The train running parameters are selected from Chinese electric multiple units high-speed train CRH3, which are shown in Table 1. Basic resistance after the piecewise linearization is presented in Figure 2, where the train speed range is from $0 \mathrm{~m} / \mathrm{s}$ to $100 \mathrm{~m} / \mathrm{s}(0-360 \mathrm{~km} / \mathrm{h})$. Through lots of experiments, we set $v_{a}=35 \mathrm{~m} / \mathrm{s}$ and $v_{b}=70 \mathrm{~m} / \mathrm{s}$ as the boundaries of piecewise linearization. The original nonlinear equations are divided into three linear equations, and the piecewise linear parameters of basic resistance are shown in Table 2.

It can be seen from Figure 2 that the running resistance of the train unit after linearization is already a good approximation to the original nonlinear curve. In fact, using more sublinear model is better for the approximation effect of the original nonlinear model, but it will increase the calculation of the controller. In order to further verify the approximate effect of the linearized train running resistance, the error of

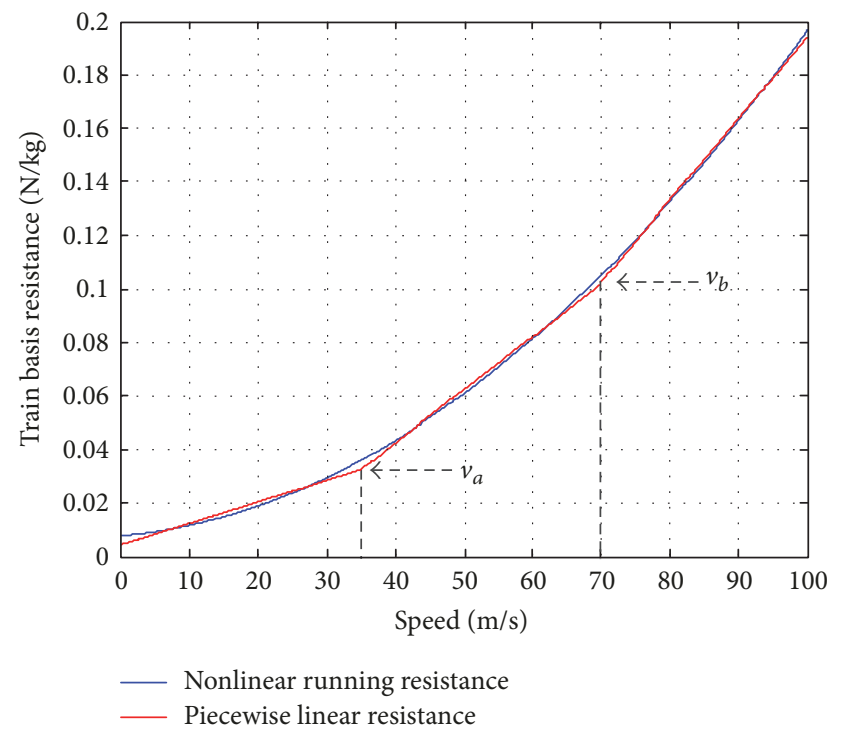

FIgURE 2: The piecewise linearization of train basic resistance.

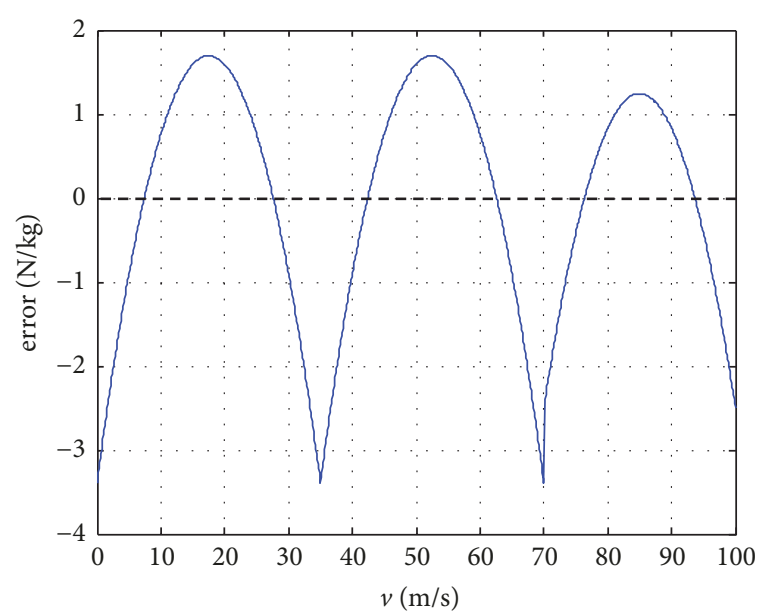

FIGURE 3: The error between the piecewise linearization of train basic resistance and the original true basic resistance.

the approximate linear curve and the original curve is drawn, which is shown in Figure 3. From Figure 3, the error between the piecewise linearization of running basic resistance and the 
practical basic resistance is less than $3.3 \mathrm{~N} / \mathrm{kg}$, which is just $0.55 \%$ compared to the biggest traction force of single vehicle, $600 \mathrm{~N} / \mathrm{kg}$

Thus, the linearization of train basic running resistance can be approximated as

$$
\omega_{0}=\alpha_{j} v+\beta_{j}, \quad j=1,2,3 .
$$

In fact, due to the force constraints of hook couplers, there is small difference among the operative velocities of vehicles. Thus, the velocity of vehicle 1 can be taken as the piecewise point of piecewise linear model. Taking it into the train multiple mass-points model, the piecewise linear dynamic model of train can be described as

$$
\begin{array}{ll}
\dot{x}(t)=A_{1}^{c} x(t)+B^{c} u(t)+f_{1}^{c} & v_{i} \leq v_{a} \\
\dot{x}(t)=A_{2}^{c} x(t)+B^{c} u(t)+f_{2}^{c} & v_{a}<v_{i} \leq v_{b} \\
\dot{x}(t)=A_{3}^{c} x(t)+B^{c} u(t)+f_{3}^{c} & v_{i}>v_{b},
\end{array}
$$

where the state variable $x=\left[v_{1}, v_{2}, \ldots, v_{n}, x_{1}, x_{2}, \ldots, x_{n}\right]^{T}$, control input $u=\left[u_{1}, u_{2}, \ldots, u_{n}\right]^{T}$, system matrix $A_{j}^{c} \in$ $\mathfrak{R}^{2 n \times 2 n}, B^{c} \in \mathfrak{R}^{2 n \times n}$, and $f_{j}^{c} \in \mathfrak{R}^{2 n \times 1}$ are as follows:

$$
\begin{aligned}
& A_{j}^{c}=\left[\begin{array}{ll}
A_{11}^{j} & A_{12}^{j} \\
I_{n \times n} & 0_{n \times n}
\end{array}\right], \\
& B^{c}=\left[\begin{array}{c}
B_{11} \\
0_{n \times n}
\end{array}\right] \text {, } \\
& f_{j}^{c}=\left[f_{j}^{1}, f_{j}^{2}, \ldots, f_{j}^{n}, 0,0, \ldots, 0\right]^{T}, \\
& A_{11}^{j}=\left[\begin{array}{ccccccc}
-\frac{d}{m_{1}}-\alpha_{j} & \frac{d}{m_{1}} & 0 & \cdots & 0 & 0 & 0 \\
\frac{d}{m_{2}} & -\frac{2 d}{m_{2}}-\alpha_{j} & \frac{d}{m_{2}} & \cdots & 0 & 0 & 0 \\
\vdots & \vdots & \vdots & \ddots & \vdots & \vdots & \\
0 & 0 & 0 & \cdots & \frac{d}{m_{n-1}} & -\frac{2 d}{m_{n-1}}-\alpha_{j} & \frac{d}{m_{n-1}} \\
0 & 0 & 0 & \cdots & 0 & \frac{d}{m_{n}} & -\frac{d}{m_{n}}-\alpha_{j}
\end{array}\right], \\
& A_{12}^{j}=\left[\begin{array}{ccccccc}
-\frac{k}{m_{1}} & \frac{k}{m_{1}} & 0 & \cdots & 0 & 0 & 0 \\
\frac{k}{m_{2}} & -\frac{2 k}{m_{2}} & \frac{k}{m_{2}} & \cdots & 0 & 0 & 0 \\
\vdots & \vdots & & \ddots & \vdots & \vdots & \vdots \\
0 & 0 & 0 & \cdots & \frac{k}{m_{n-1}} & -\frac{2 k}{m_{n-1}} & \frac{k}{m_{n-1}} \\
0 & 0 & 0 & \cdots & 0 & \frac{k}{m_{n}} & -\frac{k}{m_{n}}
\end{array}\right],
\end{aligned}
$$

and $B_{11}=\operatorname{diag}\left\{1 / m_{1}, 1 / m_{2}, \ldots, 1 / m_{n}\right\}$, and $f_{j}^{i}=k_{1} L_{e} / m_{i}-$ $\beta_{j}-9.98 \sin \theta_{i}-0.004 D_{i}$. In practical controller design, $L_{e}$ can be eliminated by state transformation, so the equilibrium position is set to zero for each subsystem.

Then, the zero-order holder is used to discretize the continuous time model. By setting the sampling period as $T_{s}$, the discrete-time piecewise linear model can be obtained as

$$
x(k+1)=A_{j}^{d} x(k)+B_{j}^{d} u(k)+f_{j}^{d}, \quad j=1,2,3,
$$

where $A_{j}^{d}=e^{A_{j}^{c} T_{s}}, B_{j}^{d}=\int_{0}^{T_{s}} e^{A_{j}^{c} \tau} d \tau B^{c}$, and $f_{j}^{d}=\int_{0}^{T_{s}} e^{A_{j}^{c} \tau} d \tau f_{j}^{c}$.

\section{Hybrid MPC Based ATO Design}

A discrete-time piecewise linear model of high-speed trains has been established, which consists of multiple piecewise linear submodels. For the traditional predictive controller design based on the piecewise linear model, the linear submodel based on the current state is determined at the beginning of the prediction and then the predictive control is operated according to the determined linear submodel. However, the predictive submodel has been fixed and does not change throughout the prediction horizon, which cannot 
reflect the system nonlinear characteristics by only using a fixed submodel. Actually, the predictive system state may change, and the corresponding submodel may also switch during the solution process. Using a fixed submodel is not enough to express the nonlinearity of the original model, especially near the switching point between submodels. Therefore, the traditional predictive control based on piecewise linear model may produce large prediction errors.

In order to address the above issues, a mixed logic dynamical system is introduced in this paper, which is proposed by Bemporad and Morari in the context of MPC technology [28]. By introducing logical variables to represent different linear submodels, the piecewise linear system can be transformed into a mixed logic dynamical system, which is a global dynamical system covering the whole nonlinear dynamics. Based on the model predictive control of the mixed logic dynamical system, the switching of the model can be expressed by the change of the logic quantity in the model. The model can be switchable throughout the predicted horizon, which ensures more accurate approximation of the original nonlinear model and reduces the prediction error. According to the propositional transformation of the mixed logic dynamic system and mixed integer linear inequality conversion rules, we transform the train piecewise linear model into a mixed integer dynamic model by introducing the binary variables [28].

Two binary variables $\delta_{1}(k)$ and $\delta_{2}(k)$ are given by

$$
\begin{aligned}
& {\left[v_{i} \leq v_{a}\right] \Longleftrightarrow\left[\delta_{1}(k)=1\right]} \\
& {\left[v_{i} \leq v_{b}\right] \Longleftrightarrow\left[\delta_{2}(k)=1\right] .}
\end{aligned}
$$

Based on $v_{i} \in\left[v_{\min }, v_{\max }\right]$, (9) can be written as follows:

$$
\begin{aligned}
& \delta_{1}(k)\left(v_{a}-v_{\min }\right) \geq v_{i}(k)-v_{\min } \\
& \delta_{1}(k)\left(v_{a}-v_{\max }\right) \leq v_{a}-v_{i}(k)-\varepsilon \\
& \delta_{2}(k)\left(v_{b}-v_{\min }\right) \geq v_{i}(k)-v_{\min } \\
& \delta_{2}(k)\left(v_{b}-v_{\max }\right) \leq v_{b}-v_{i}(k)-\varepsilon,
\end{aligned}
$$

where $\varepsilon$ is a small positive number and generally takes $10^{-6}$. It is used to transform a strict equality into a nonstrict inequality.

By defining $\Phi_{i}(k)=A_{i}^{d} x(k)+B_{i}^{d} u(k)+f_{i}^{d}$, the relationship between $\delta_{1}(k), \delta_{2}(k)$, and $x(k+1)$ can be shown in Table 3 .

Then, the piecewise linear system (8) can be described as

$$
\begin{aligned}
x(k+1)= & \delta_{1}(k) \delta_{2}(k) \Phi_{1}(k) \\
& +\left(1-\delta_{1}(k)\right) \delta_{2}(k) \Phi_{2}(k) \\
& +\left(1-\delta_{1}(k)\right)\left(1-\delta_{2}(k)\right) \Phi_{3}(k) .
\end{aligned}
$$

We introduce a new variable $\delta_{3}(k)=\delta_{1}(k) \delta_{2}(k)$, which satisfies

$$
\begin{array}{r}
-\delta_{1}(k)+\delta_{3}(k) \leq 0 \\
-\delta_{2}(k)+\delta_{3}(k) \leq 0 \\
\delta_{1}(k)+\delta_{2}(k)-\delta_{3}(k) \leq 1 .
\end{array}
$$

TABLE 3: The relationship between $\delta_{1}(k), \delta_{2}(k)$, and $x(k+1)$.

\begin{tabular}{lcc}
\hline$\delta_{1}(k)$ & $\delta_{2}(k)$ & $x(k+1)$ \\
\hline 1 & 1 & $\Phi_{1}(k)$ \\
0 & 1 & $\Phi_{2}(k)$ \\
0 & 0 & $\Phi_{3}(k)$ \\
\hline
\end{tabular}

In addition, the auxiliary mixed logical vectors and variables are introduced, that is, $z_{1}^{x}(k)=\delta_{1}(k) x(k), z_{2}^{x}(k)=$ $\delta_{2}(k) x(k), z_{3}^{x}(k)=\delta_{3}(k) x(k), z_{1}^{u}(k)=\delta_{1}(k) u(k), z_{2}^{u}(k)=$ $\delta_{2}(k) u(k)$, and $z_{3}^{u}(k)=\delta_{3}(k) u(k)$, which can be expressed as

$$
\begin{gathered}
x_{\min } \delta_{i}(k) \leq z_{i}^{x}(k) \leq x_{\max } \delta_{i}(k) \\
x(k)-x_{\max }\left(1-\delta_{i}(k)\right) \leq z_{i}^{x}(k) \\
\quad \leq x(k)-x_{\min }\left(1-\delta_{i}(k)\right) \\
u_{\min } \delta_{i}(k) \leq z_{i}^{u}(k) \leq u_{\max } \delta_{i}(k) \\
u(k)-u_{\max }\left(1-\delta_{i}(k)\right) \leq z_{i}^{u}(k) \\
\leq u(k)-u_{\min }\left(1-\delta_{i}(k)\right),
\end{gathered}
$$

for $i=1,2,3$, where $x \in\left[x_{\min }, x_{\max }\right]$ and $u \in\left[u_{\min }, u_{\max }\right]$. The maximum tract input $u_{\max }$ is a nonincreasing function of speed, which is approximated by a piecewise linear, quadratic, and/or hyperbolic function of speed [15]. Thus it can be described as a group of hyperbolic or parabolic formulas and each formula approximates the actual traction force for a certain speed interval [30], such as

$$
u_{\max }(v)=c_{1, j}+c_{2, j} v+c_{3, j} v^{2}, \quad v \in\left[v_{j}, v_{j+1}\right]
$$

or

$$
\begin{aligned}
& u_{\max }(v)=\frac{c_{h, j}}{v}, \\
& \qquad v \in\left[v_{j}, v_{j+1}\right], \text { for } j=1,2, \ldots, M-1,
\end{aligned}
$$

where the piecewise point $v_{j}$ and $v_{j+1}$ and the parameters $c_{1 j}, c_{2 j}, c_{3 j}$, and $c_{h j}$ can be determined by train operation experiments.

Then (8) can be transformed into the following mixed logical dynamical (MLD) system:

$$
\begin{aligned}
x(k+1)= & A x(k)+B_{1} u(k)+B_{2} \delta(k) \\
& +B_{3} z(k)+B_{4} \\
E_{2} \delta(k)+E_{3} z(k) \leq & E_{1} u(k)+E_{4} x(k)+E_{5},
\end{aligned}
$$

where $z(k)=\left[z_{1}^{x}(k)^{T} z_{2}^{x}(k)^{T} z_{3}^{x}(k)^{T} z_{1}^{u}(k) z_{2}^{u}(k) z_{3}^{u}(k)\right]^{T}$, $\delta(k)=\left[\begin{array}{lll}\delta_{1}(k) & \delta_{2}(k) & \delta_{3}(k)\end{array}\right]^{T}, A=A_{3}^{d}, B_{1}=B_{3}^{d}, B_{4}=f_{3}^{d}$, $B_{2}=\left[-f_{3}^{d}, f_{2}^{d}-f_{3}^{d}, f_{3}^{d}-f_{2}^{d}+f_{1}^{d}\right]$, and $B_{3}=\left[-A_{3}^{d}, A_{2}^{d}-\right.$ $\left.A_{3}^{d}, A_{1}^{d}-A_{2}^{d}+A_{3}^{d},-B_{3}^{d}, B_{2}^{d}-B_{3}^{d}, B_{1}^{d}-B_{2}^{d}+B_{3}^{d}\right]$.

Specifically, the resulting MLD model (14) is obtained by using the HYSDEL compiler [31]. Matrices $E_{1}, \ldots, E_{5}$ include constraints (16). They can be obtained and analyzed in Matlab using, for example, the Hybrid Toolbox [32]. 


\section{Controller Design}

Based on the MLD system model, a hybrid MPC framework is introduced in this section. Within this, the MLD model is used as the prediction model. Then the control problem is formulated as a constrained finite time optimal control problem and converted into mixed integer linear programming (MILP) problem, for which efficient solvers are available.

4.1. Formulation of Optimal Control Problem. Automatic train control needs to track the desired velocity profile quickly such that the timeliness of the interstation travel can be ensured. With the development of high-speed railway, energy-saving driving has become an important goal of automatic train control. As the running distance of the train between stations is fixed, the energy consumption directly related to the traction and braking force. Thus, the control input is used to express energy consumption through this paper. In addition, the in-train force should be kept in the desired range to ensure the safety of the train.

Therefore, the optimal problem can be established as follows, which considers the speed tracking error, control input, and the in-train force.

$$
\begin{aligned}
J\left(u_{N}(k)\right)= & \sum_{i=0}^{N-1}\|\varepsilon((k+i) \mid k)\|_{\mathrm{Q}}^{1}+\|u((k+i) \mid k)\|_{R}^{1} \\
& +\left\|x_{i n}((k+i) \mid k)\right\|_{H}^{1},
\end{aligned}
$$

where $k$ is the current sampling time; $u_{N}(k)=[u(k), \ldots, u(k+$ $N-1)]$ is the sequence of $N$ control inputs. $\varepsilon((k+i) \mid k)=$ $v((k+i) \mid k)-v^{r}(k+i) \in \mathbb{R}^{n \times 1}$ represents the difference between actual speed $v$ and reference speed $v^{r}, u((k+i) \mid k)=$ $\left[u_{1}(k+i), u_{2}(k+i), \ldots, u_{n}(k+i)\right]^{T}$ represents the control input, and $x_{i n}((k+i) \mid k)=\left(x_{i n}^{1}\left(((k+i) \mid k), \ldots, x_{i n}^{n-1}(((k+i) \mid k))\right.\right.$ represents the relative displacements between neighboring cars. 1 means 1 -norm and $Q, R$, and $H$ are the weight matrixes, respectively.

In order to ensure the safe and efficient operation of the train, some constraints must be satisfied as follows.

(1) Constraint on Control Input. The tractive and braking forces are bounded due to the nature physical characteristics of the traction motor. Thus, the control input is set as $-F_{\max }^{B} \leq$ $u_{i}(k) \leq F_{\max }^{T}$.

(2) Constraint on Maximum Operational Velocity. The maximum speed of the train depends first on the maximum speed allowed by the train itself. Second, when the line conditions and operating conditions change, the maximum allowable speed of the train may change.

(3) Constraint on In-Train Force. The in-train force should be in the range $\left[-f_{\max }^{\text {in }}, f_{\max }^{\text {in }}\right]$ to keep the operational safety of the trains.

(4) Constraint on Coupler Deformation. The coupler deformation should be in the range $\left[-\Delta L_{\max }, \Delta L_{\max }\right]$ to keep the operational safety of the trains.
Then, the constraints considered can be formulated by

$$
\begin{array}{r}
-F_{\max }^{B} \leq u_{i}(k) \leq F_{\max }^{T} \\
0 \leq v_{i}(k) \leq v_{\max } \\
-f_{\max }^{i n} \leq f_{i, i+1}^{i n} \leq f_{\max }^{\text {in }} \\
-\Delta L_{\max } \leq \Delta L_{i} \leq \Delta L_{\max } .
\end{array}
$$

4.2. Hybrid MPC Controller Design. Based on (17) and (18), a constrained optimal control problem can be constructed as follows:

$$
\begin{array}{ll}
\min & J\left(u_{N}(k)\right) \\
= & \sum_{i=0}^{N-1}\|\varepsilon((k+i) \mid k)\|_{Q}^{1}+\|u((k+i) \mid k)\|_{R}^{1} \\
& +\left\|x_{\text {in }}((k+i) \mid k)\right\|_{H}^{1} \\
\text { s.t. } \quad & x(k+1) \\
& =A x(k)+B_{1} u(k)+B_{2} \delta(k)+B_{3} z(k)+B_{4} \\
& E_{2} \delta(k)+E_{3} z(k) \leq E_{1} u(k)+E_{4} x(k)+E_{5} \\
& -F_{\max }^{B} \leq u_{i}(k) \leq F_{\max }^{T} \\
& 0 \leq v_{i}(k) \leq v_{\max } \\
& -f_{\max }^{\text {in }} \leq f_{i, i+1}^{\text {in }} \leq f_{\max }^{\text {in }} \\
& -\Delta L_{\max } \leq \Delta L_{i} \leq \Delta L_{\max } .
\end{array}
$$

In order to facilitate the solution of the optimal problem, (19) can be transformed to the linear objective function (20) by introducing the auxiliary variables $w^{\varepsilon}(k+i \mid k)=\| \varepsilon((k+i) \mid$ $k)\left\|_{Q}^{1}, w^{u}(k+i \mid k)=\right\| u((k+i) \mid k) \|_{R}^{1}$, and $w^{x_{\text {in }}}(k+i \mid k)=$ $\left\|x_{\text {in }}((k+i) \mid k)\right\|_{H}^{1}$.

$$
\begin{aligned}
J\left(u_{N}(k)\right)= & w^{\varepsilon}(k+N \mid k)+\sum_{i=0}^{N-1} w^{\varepsilon}(k+i \mid k) \\
& +w^{u}(k+i \mid k)+w^{x_{i n}}(k+i \mid k)
\end{aligned}
$$

with some linear inequalities:

$$
\begin{gathered}
w^{\varepsilon}(k+i \mid k) \geq \pm \varepsilon((k+i) \mid k) \\
w^{u}(k+i \mid k) \geq \pm u((k+i) \mid k) \\
w^{x_{i n}}(k+i \mid k) \geq \pm x_{i n}((k+i) \mid k) .
\end{gathered}
$$

In addition, the state update equation (22) can be obtained by the MLD model.

$$
\begin{gathered}
x(k+i)=A^{i} x(k)+\sum_{j=0}^{i-1} A^{j}\left(B_{1} u(k+i-j-1)\right. \\
\left.+B_{2} \delta(k+i-j-1)+B_{3} z(k+i-j-1)\right) \\
E_{2} \delta(k)+E_{3} z(k) \leq E_{1} u(k)+E_{4} x(k)+E_{5} .
\end{gathered}
$$


TABLE 4: Definition of some notations.

\begin{tabular}{lc}
\hline Notation & Definition \\
\hline$P$ & The MILP problem \\
$J_{0}^{*}$ & The minimum upper bound of optimal performance of train operation \\
$V^{*}$ & The optimal solution vector of optimal performance index for train operation \\
$\Pi$ & The object table of recording all LP subproblems that need to be solved \\
$J(\bar{V})$ & The optimal performance of the current relaxed LP problem \\
$\bar{V}$ & The optimal solution vector of the relaxed LP subproblem \\
$Q_{\text {num }}$ & Number of LP subproblems that have been solved \\
Maxnum $\operatorname{LP}(j)\left[P_{0}\right], \operatorname{LP}(j)\left[P_{1}\right]$ & The maximum number of LP problems \\
\hline
\end{tabular}

Then, based on (20), (21), and (22), the optimal control problem can be converted to a mixed integer linear programming (MILP) problem as follows:

$$
\begin{aligned}
J(V, \theta)=\min _{V} & C^{T} V \\
\text { st. } & G V \leq W+S \theta(k),
\end{aligned}
$$

where $\theta(k)=\left[x(k)^{T}, v^{r}(k), \ldots, v^{r}\left(k+N_{p}-1\right)\right]^{T}$ is commonly represented as the parameter vector and $V=$ $\left[u_{N_{p}}^{\prime}(k), \widetilde{\delta}(k)^{T}, \widetilde{z}(k)^{T}, \widetilde{w}(k)^{T}\right]^{T}$ is the optimization vector. Let

$$
\begin{aligned}
& \widetilde{\delta}(k)=\left[\begin{array}{c}
\delta(k) \\
\delta(k+1) \\
\vdots \\
\delta\left(k+N_{p}-1\right)
\end{array}\right], \\
& \widetilde{z}(k)= {\left[\begin{array}{c}
z(k) \\
z(k+1) \\
\vdots \\
z\left(k+N_{p}-1\right)
\end{array}\right], } \\
& \widetilde{w}(k)=\left[w^{\varepsilon}(k), w^{u^{\prime}}(k), \ldots, w^{\varepsilon}\left(k+N_{p}-1\right),\right. \\
&\left.w^{u^{\prime}}\left(k+N_{p}-1\right)\right]^{T} ;
\end{aligned}
$$

$C^{T}=[0, \ldots, 0,1, \ldots, 1]$. The matrices $G, W$, and $S$ are obtained according to (16), (18), and (21).

At each sampling step $k$, the MILP problem is solved based on the current parameter vector $\theta(k)$, and an optimal sequence of future control input $u_{N_{p}}^{\prime}(k)$ can be obtained. At time $k+1$, the parameter vector is updated to $\theta(k+1)$, and a new MILP problem is solved to get the new optimal control input. This procedure is repeated at each sampling step, and the problems can be solved in real time. In this paper, only $N_{c}=N_{p}$ is considered.

4.3. Solution to MILP Problem. The problem of automatic train control is established as a constrained finite time optimal control problem and further transformed into mixed integer linear programming (MILP) problem by analyzing the operation target and constraints. Since the constructed MILP problem contains logical variables and continuous variables, this section uses the branch and bound method $(B \& B)$ to solve this problem so that, in each control cycle, the optimal traction of each vehicle or braking force output can be obtained.

The basic idea of branch and bound method is to relax the logic variable constraints in the optimization problem. $\delta_{i}(k) \in\{0,1\}$ in the MLD model of formula (16) can be relaxed as $\delta_{i}(k) \in[0,1]$, which transforms the original MILP problem into linear programming (LP). Then, the traditional linear programming method is used to solve the optimal subproblem. Algorithm 1 is presented to solve the MILP problem and some notations are defined and presented in Table 4.

\section{Performance Evaluation}

In this section, we will evaluate the performance of the proposed hybrid MPC ATO algorithm using numerical results. We will first introduce the parameter settings of the ATO system. Specifically, the parameters of the train model are from the CRH-3 high-speed train in China, which are given in Table 5. Therein, $F_{\max }^{T}$ and $F_{\max }^{B}$ are the maximum tractive force and braking force, respectively.

5.1. Simulation Scenario. In order to evaluate the performance of the controller, the desired velocity curve including accelerating, decelerating, velocity step increase, velocity step decrease, and constant-velocity stages is characterized by the following equation:

$$
v_{r}(t)= \begin{cases}0.4 t & 0 \leq t \leq 100 \\ 40 & 100 \leq t \leq 400 \\ 75 & 400 \leq t \leq 900 \\ 50 & 900 \leq t \leq 1150 \\ -t+1200 & 1150 \leq t \leq 1200\end{cases}
$$

In (25), the acceleration is $0.4 \mathrm{~m} / \mathrm{s}$, and the deceleration is $0.5 \mathrm{~m} / \mathrm{s}$, both of which are within the threshold of acceleration and velocity. In the operation of trains, the basic resistance is the main resistance for the trains. Thus, we only consider the basic resistance in this paper. 


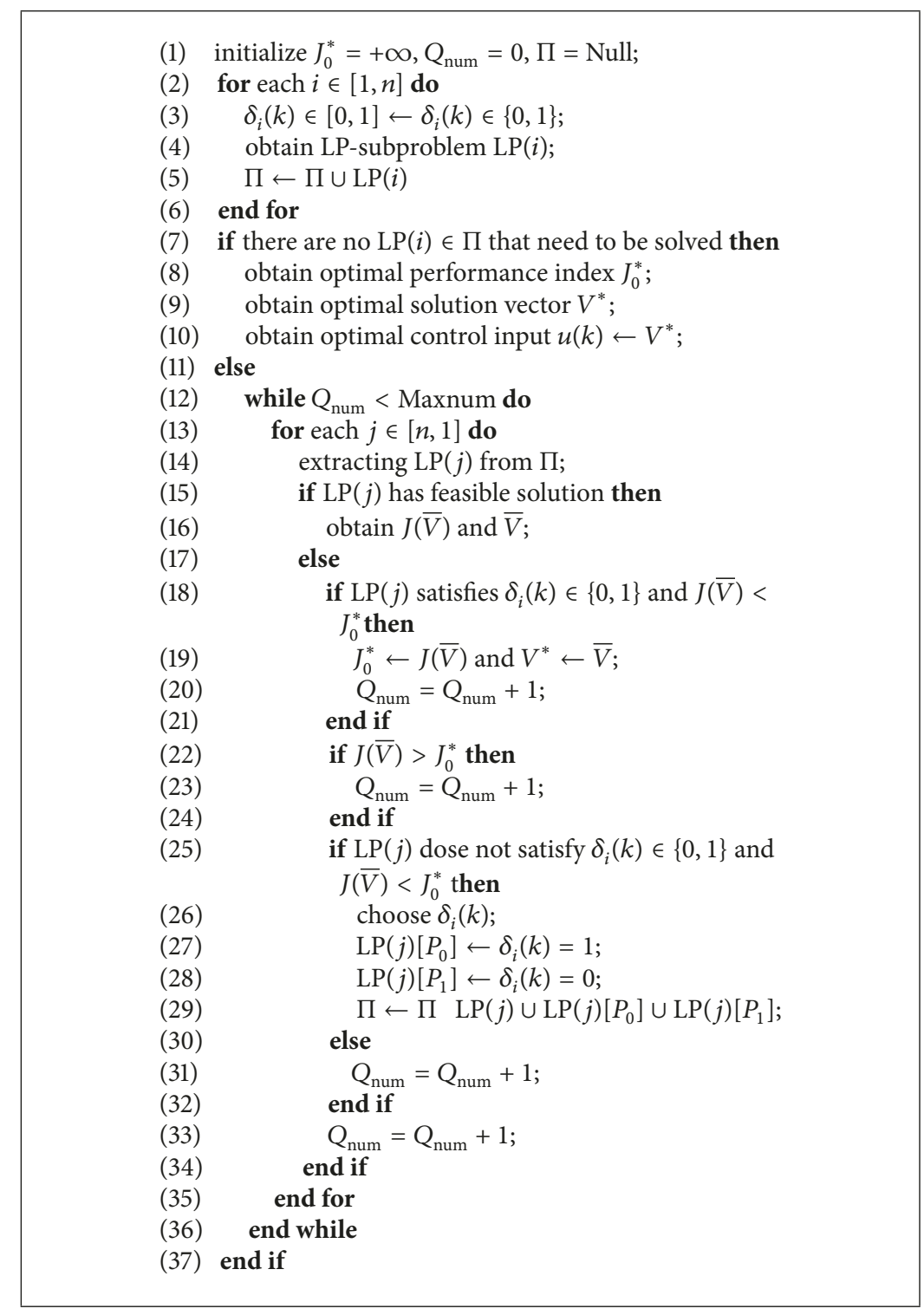

Algorithm 1: Solution of MILP problem.

The designed MPC based ATO controller considers the train velocity tracking performance, energy consumption, and intertrain force. With the train parameters, we provide two simulation scenarios to evaluate the performance of the proposed algorithm under different operating conditions. Specifically, we evaluate the performance of the controller with different train types: all-locomotive train and partiallocomotive train.

5.1.1. Scenario A. In this scenario, we evaluate the performance of the ATO controller with the all-locomotive train. To simplify the simulation, we choose the number of trains as $n=4$, and the train is comprised of all locomotives. This implies that each locomotive has the tracking motor and braking unit and thus can provide both the tracking force and the braking force. The parameters of the train are shown in Table 5, where $m_{i}$ is the mass of locomotive $i$. Since the numbers of passengers in each locomotive may be different, we choose $m_{1}=48.5 t, m_{2}=52.5 t, m_{3}=51.0 t, m_{4}=47.5 t$. For the MPC controller, the prediction horizon $N_{p}$ and the control horizon $N_{c}$ also affect the controller performance. Without loss of generality, we choose $N_{p}=N_{c}=3$. The weighting matrix gain is chosen as

$$
\begin{aligned}
& Q=\operatorname{diag}(\underbrace{4, \ldots, 4}_{n}), \\
& R=\operatorname{diag}(\underbrace{1, \ldots, 1}_{n}), \\
& H=\operatorname{diag}(\underbrace{1, \ldots, 1}_{n-1}) .
\end{aligned}
$$

5.1.2. Scenario B. In this scenario, we evaluate the effectiveness of the ATO controller in partial-locomotive trains. To 
TABLE 5: Parameters of the CRH-3 high-speed train.

\begin{tabular}{|c|c|c|}
\hline Parameter & Value & Unit \\
\hline$m_{i}$ & 47.5 & $\mathrm{t}$ \\
\hline$c_{0}$ & $7.75 \times 10^{-3}$ & $\mathrm{Nkg}^{-1}$ \\
\hline$c_{v}$ & $2.28 \times 10^{-4}$ & $\mathrm{Ns}(\mathrm{mkg})^{-1}$ \\
\hline$c_{a}$ & $1.66 \times 10^{-5}$ & $\mathrm{Ns}^{2}\left(\mathrm{~m}^{2} \mathrm{~kg}\right)^{-1}$ \\
\hline$F_{\max }^{T}$ & 300 & $\mathrm{kN}$ \\
\hline$F_{\max }^{B}$ & 300 & $\mathrm{kN}$ \\
\hline$k$ & $1 \times 10^{4}$ & $\mathrm{kNm}^{-1}$ \\
\hline$d$ & 1000 & $\mathrm{kNsm}^{-1}$ \\
\hline$\theta$ & 0 & - \\
\hline$D$ & 0 & - \\
\hline$\Delta L_{\max }$ & 0.2 & $\mathrm{~m}$ \\
\hline$f_{\max }^{i n}$ & 2000 & $\mathrm{kN}$ \\
\hline
\end{tabular}

simplify the simulation, we choose the number of trains as $n=4$, and the train is comprised of 2 locomotives and 2 wagons. The locomotive can provide both the tracking force and the braking force, while the wagons can only supply the brake forces. In order to guarantee the total force the same as Scenario $\mathrm{A}$, the maximum tracking force of each locomotive is set as $F_{\max }^{T}=600 \mathrm{kN}$, and the other train parameters are the same as in Table 5.

5.2. Simulation Results. We will provide simulation results of the ATO controller in different operating conditions. The simulations are conducted in Matlab/SIMULINK. We fist consider the simulations in Scenario A, which is based on all-locomotive train. Then we will further consider the simulation results in Scenario B, which is based on partiallocomotive trains. And in Scenario $\mathrm{C}$, we focus on more realistic condition where the track force limit is dynamic.

5.2.1. Scenario $A$. The velocity curve of each locomotive is shown in Figure 4, where $x$-axis represents the time and $y$ axis represents the velocity. From the figure, we can see that the velocities of four locomotives almost remain the same during the operation time. Specifically, in the accelerating and decelerating stages, each locomotive can operate correspondingly according to the given acceleration and deceleration. At $t=400 \mathrm{~s}$, we notice that the reference velocity has the step increase. From the zoomed-in figure, we can find that the locomotives move with the almost same velocity, where the maximum velocity error is $0.003 \mathrm{~m} / \mathrm{s}$. From the zoomed-in figure from $t=470 \mathrm{~s}$ to $t=490 \mathrm{~s}$, the velocities of locomotives reach the steady value at about $t=490 \mathrm{~s}$, where the settling time is $90 \mathrm{~s}$ and the steady error is $0.5 \mathrm{~m} / \mathrm{s}$. In the steady state, the velocities of locomotives remain fixed. Based on the simulation results, we can find that the train can track the time-varying reference with small settling time and steady error, which verifies the effectiveness of the algorithm.

The tracking and braking forces of locomotives are shown in Figure 5. In the accelerating stage, the resistance of the train increases with the velocity. In order to guarantee the constant acceleration, the tracking forces of locomotives increase gradually. In the deceleration stage, the resistance of

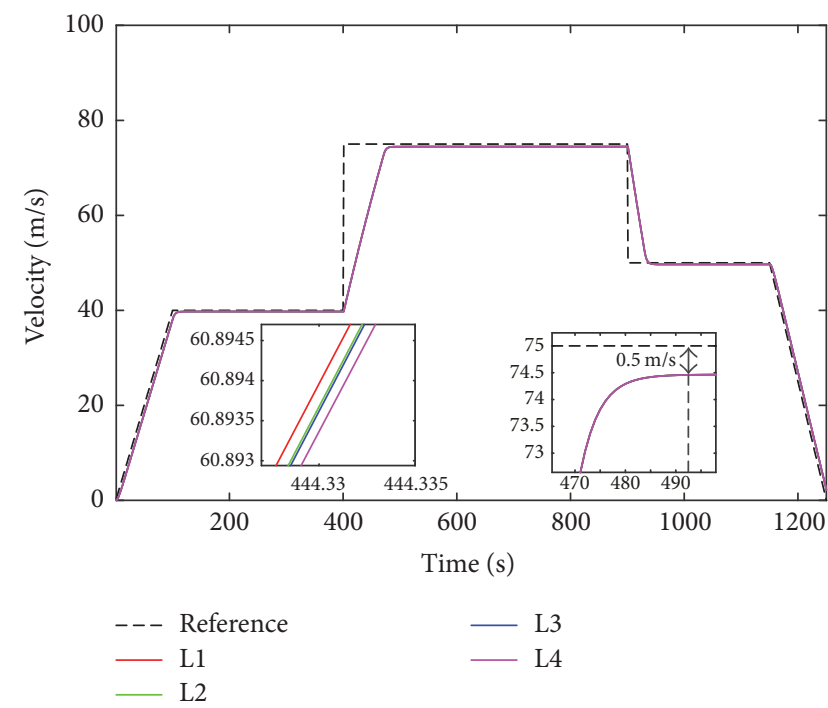

FIgURE 4: The velocity curve of each locomotive in Scenario A.

the train decelerates with the velocity. In order to guarantee the constant deceleration, the braking forces of locomotives increase gradually. Compared with the acceleration or deceleration stages, the tracking forces of locomotives are small and fixed during the constant velocity stage. This is because the resistance of train keeps having a relatively small value during the constant velocity stage. At $t=400 \mathrm{~s}$, the reference has a step increase. In order to accelerate to the desired velocity, the tracking forces of locomotives increase to the maximum rapidly and maintain the maximum tracking force for about $72 \mathrm{~s}$. At $t=900 \mathrm{~s}$, the braking forces increase to the maximum to decrease the velocity and maintain about $32 \mathrm{~s}$ to decrease to the desired velocity. From the figure, we can find that the control outputs of locomotives are almost the same and thus guarantee the reasonable allocation of braking force and tracking force. When the reference velocity curve changes, each locomotive can regulate the tracking force and braking force based on the difference between the actual velocity and desired velocity. The forces for locomotives are 


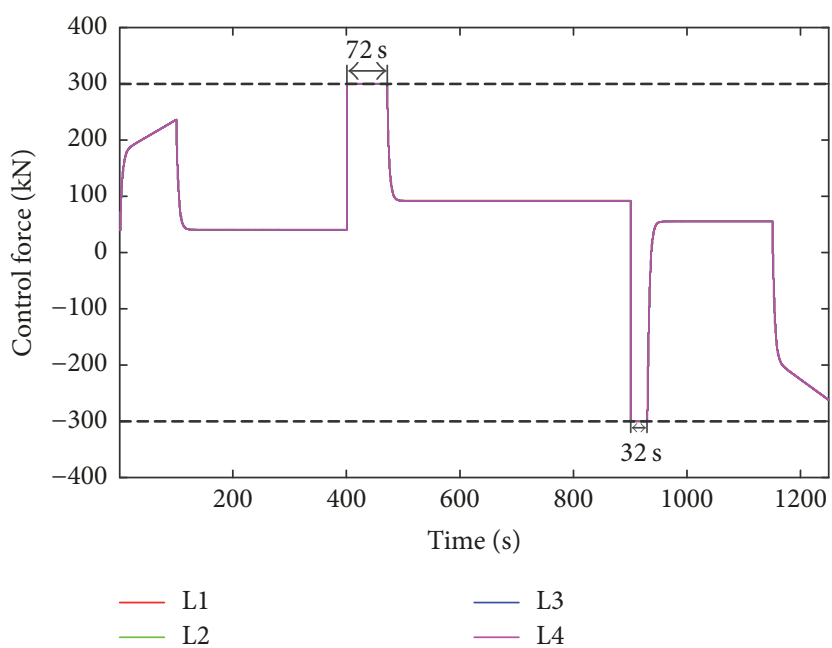

Figure 5: The force output of each locomotive in Scenario A.
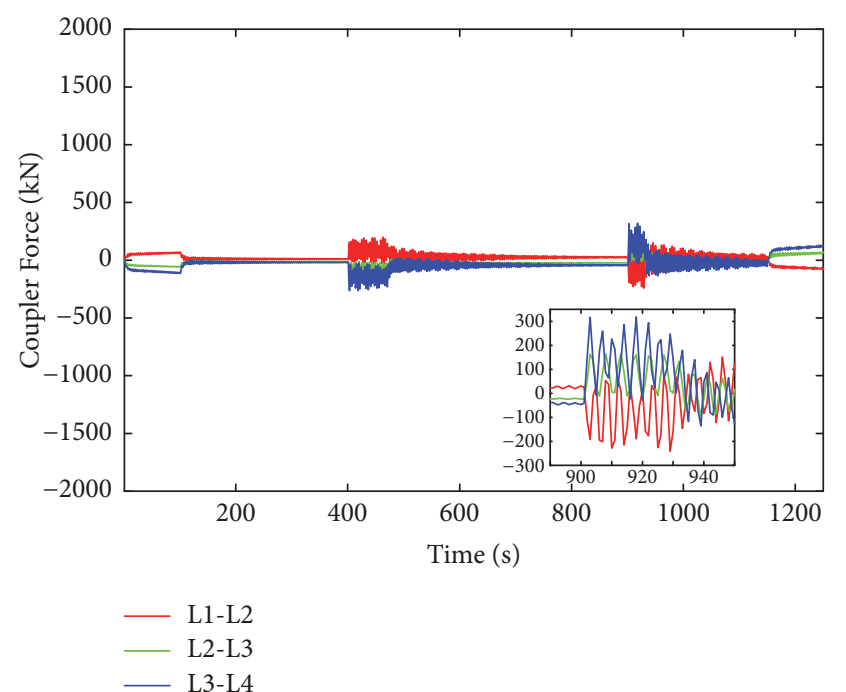

FIgURE 6: The coupler force among locomotives in Scenario A.

almost the same and also satisfy the constraints, which verify the effectiveness of the proposed strategy.

The coupler force among each locomotive is shown in Figure 6. When the coupler force is larger than zero, it means that coupler is pulled. When the coupler force is smaller than zero, the coupler is pushed. From the figure, we can see that the coupler forces among locomotives are always within the safety range. Moreover, the coupler among L1 and L2 is mainly pulled, while the coupler among L3 and L4 is mainly pushed. This is because when we choose the masses of locomotives, the masses of L3 and L4 are relatively smaller than L1 and L2. In order to guarantee the velocity consensus of locomotives, the total forces should be the same and the coupler force can balance the total force of each locomotive. It can be found that, during the constant-velocity stage, constant-accelerating stage, and constant-decelerating stage, the coupler force among locomotives is small. At $t=$ $200 \mathrm{~s}$ and $t=400 \mathrm{~s}$, the coupler force changes dramatically,

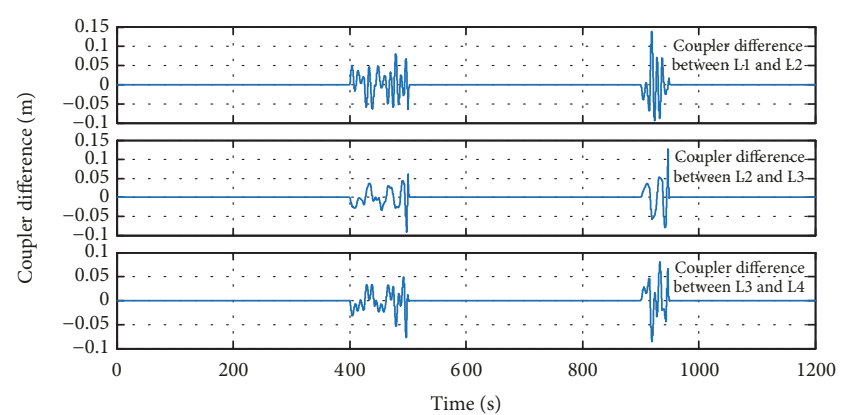

Figure 7: The coupler deformation between two neighbouring locomotives in Scenario A.

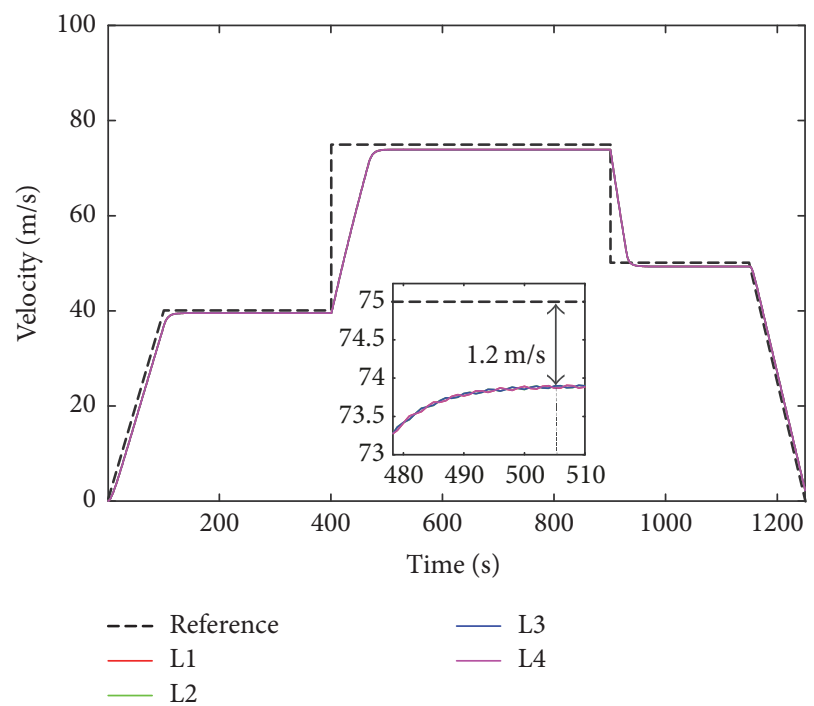

Figure 8: The velocity curve of each vehicle in Scenario B.

with the largest coupler pulling force $317 \mathrm{kN}$ and the largest coupler pushing force $241 \mathrm{kN}$. From the figure, we can see that the ATO controller can guarantee that the coupler force is within the safety range, which verifies the effectiveness.

The operation safety of high-speed trains can be evaluated by the position difference (coupler deformation) between neighboring locomotives. If the deformation is larger than the maximum $0.2 \mathrm{~m}$ or less than the minimum $-0.2 \mathrm{~m}$, the coupler may have been broken so the safety cannot be guaranteed. The operation safety of high-speed trains can be further illustrated from Figure 7, which shows that the coupler deformation is always with the threshold $[-0.2 \mathrm{~m}, 0.2 \mathrm{~m}]$. This is because the in-train force guarantees that the position difference between neighboring locomotives cannot be too large or too small.

5.2.2. Scenario B. The simulation in Scenario A verifies the effectiveness of the hybrid model predictive controller for all-locomotive case. The metrics include velocity tracking, energy consumption, and coupler force.

In Scenario B, we verified the effectiveness of the ATO controller with 2 locomotives and 2 wagons. The velocity curves are shown in Figure 8. With the same controller 

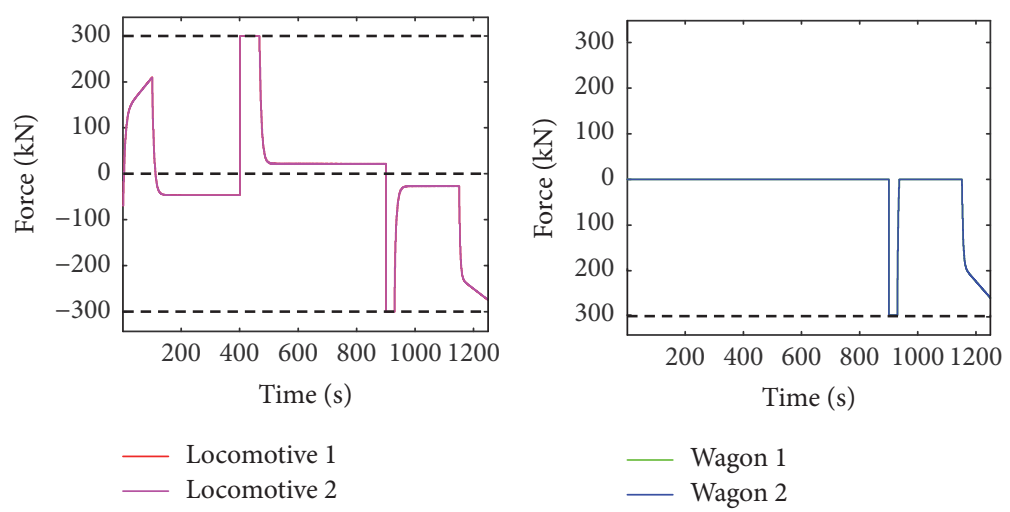

FIGURE 9: The forces of locomotives and wagons in Scenario B.

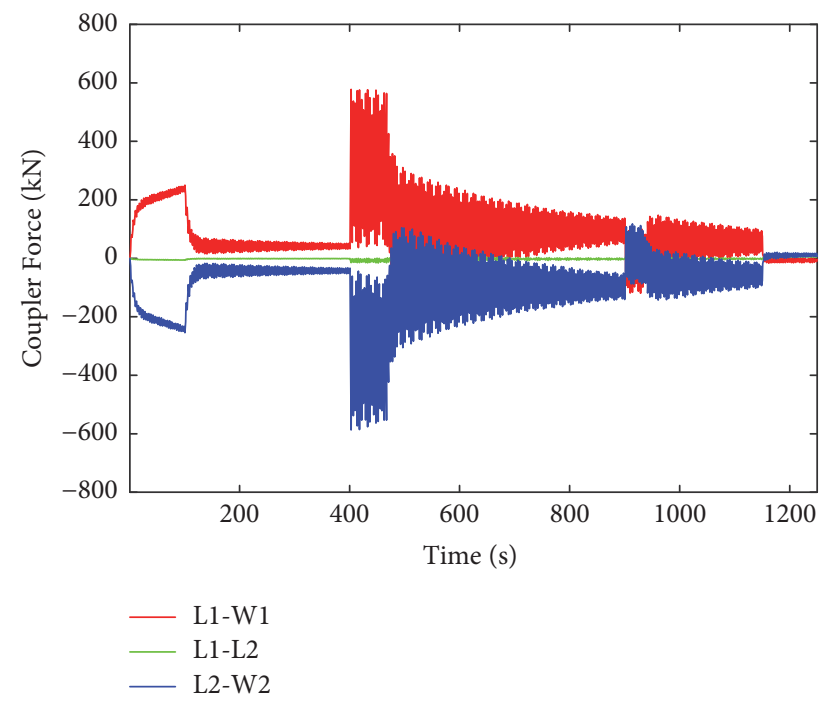

FIgURE 10: The coupler force among vehicles in Scenario B.

parameters, at $t=400 \mathrm{~s}$, where the reference velocity has a step change, the convergence time of the controller from the change at $t=400 \mathrm{~s}$ to the steady state at $t=508 \mathrm{~s}$ implies that the convergence time increases by $18 \mathrm{~s}$ compared with the all-locomotive case. The steady error is $1.2 \mathrm{~m} / \mathrm{s}$, which increases by $0.7 \mathrm{~m} / \mathrm{s}$ compared with the all-locomotive case. Moreover, the velocities at the steady stage have some oscillations around the steady value.

The force output of each vehicle is shown in Figure 9, where the left subfigure shows the force output of the locomotive, and the right subfigure shows the force output of the wagon. From the figure, we can see that, during the acceleration and constant-velocity stages, only the locomotive has the tracking force output, and the tracking force of wagons is zero. In the deceleration stage, both the locomotives and wagons supply the braking forces.

Figure 10 shows the coupler force among vehicles. Compared with the all-locomotive trains, the coupler forces between L1 and W1 and between L2 and W2 are kept at large values. This is because locomotive $\mathrm{L} 1$ supplies the pulling force and locomotive L2 supplies the pushing force, while

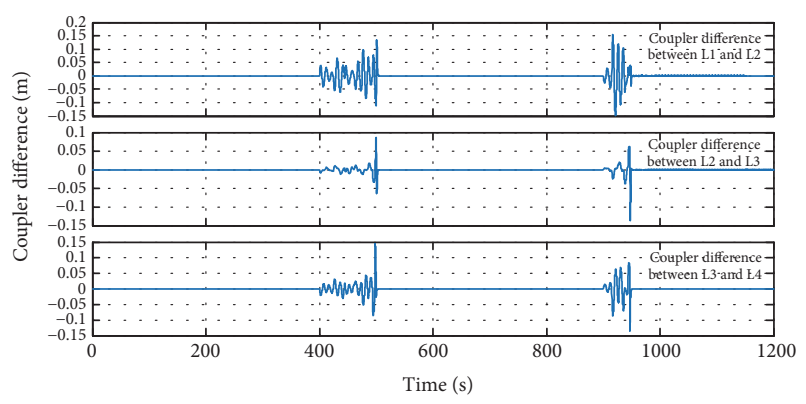

FIGURE 11: The coupler deformation between neighboring locomotives/wagons in Scenario B.

wagons W1 and W2 cannot supply the tracking force. The maximum coupler force is $578 \mathrm{kN}$, which is within the safety threshold. From the above simulations, we can find that the proposed hybrid MPC ATO controller has a good control effect.

The operation safety of high-speed trains can be illustrated from Figure 11, which depicts the spring deformation between neighboring locomotives/wagons. We can see that the largest deformation is $0.14 \mathrm{~m}$, and the smallest deformation is $-0.15 \mathrm{~m}$, which is with the safety threshold $[-0.2 \mathrm{~m}, 0.2 \mathrm{~m}]$. As illustrated in Figure 7, this is because the in-train force guarantees that the position difference between neighboring locomotives cannot be too large or too small. Simulation results show that the proposed ATO controller can guarantee the safe operation of high-speed trains.

5.3. Further Discussions. In this subsection, we further discuss the performance of the proposed ATO controller in terms of practicality, computation efficiency, superiority, and robustness. For simplicity, we only consider Scenario A, where the train is comprised of four locomotives. Scenario $\mathrm{B}$ can also be analyzed similarly. The train parameters are set the same as in Table 5, unless otherwise specified.

5.3.1. Practicality. In the previous simulation setup, we assume that the maximum traction force $F_{\max }^{T}$ and the maximum braking force $F_{\max }^{B}$ are constants. Despite simplifying the implementation, the constant maximum traction/braking 
TABLE 6: Computational time (s) of each MPC step with and without linearization.

\begin{tabular}{lccccc}
\hline & \multicolumn{3}{c}{ MPC with linearization } & \multicolumn{2}{c}{ MPC without linearization } \\
& Min. & Max. & Average & Min. & Max. \\
\hline$N_{c}=3$ & $1.6 e-3$ & $3.8 e-3$ & $2.5 e-3$ & $2.1 e-1$ & $3.6 e-1$ \\
$N_{c}=5$ & $1.8 e-3$ & $6.4 e-3$ & $3.5 e-3$ & $2.5 e-1$ & $8.3 e-1$ \\
$N_{c}=7$ & $1.9 e-3$ & $7.3 e-3$ & $4.6 e-3$ & $3.9 e-1$ & $9.8 e-1$ \\
\hline
\end{tabular}

force are not realistic as the operation is subject to the power limit of the train. This implies that the maximum traction and braking forces evolve with the operation speed; that is, the maximum traction and braking forces for the CRH-3 train are represented as in [33].

$$
\begin{aligned}
& F_{\max }^{T}= \begin{cases}300-0.284 v, & 0 \leq v \leq 119.7 \mathrm{~km} / \mathrm{h} \\
266 \times \frac{119.7}{v}, & 119.7<v \leq 300 \mathrm{~km} / \mathrm{h}\end{cases} \\
& F_{\text {max }}^{B}= \begin{cases}300-0.281 v, & 0 \leq v \leq 106.7 \mathrm{~km} / \mathrm{h} \\
266 \times \frac{119.7}{v}, & 106.7<v \leq 300 \mathrm{~km} / \mathrm{h} .\end{cases}
\end{aligned}
$$

The force output of each locomotive with power limit is shown in Figure 12, where we find that the output force of each locomotive evolves with time. It is interesting to note that at $t=400 \mathrm{~s}$ the maximum traction force decreases at the transition stage, where the speed of the train increases from $40 \mathrm{~m} / \mathrm{s}$ to $75 \mathrm{~m} / \mathrm{s}$. Due to the constant-power operation, the maximum traction force will decrease with the increase of velocity. At $t=900 \mathrm{~s}$, it is shown that the maximum braking force increases when the velocity decreases from $75 \mathrm{~m} / \mathrm{s}$ to $50 \mathrm{~m} / \mathrm{s}$.

5.3.2. Computational Time. In this subsection, we consider the computation time of the proposed method with different prediction horizons.

Within different control horizons, the nonlinear MPC and the proposed piecewise MPC are both implemented on the original nonlinear system. The simulations are performed under Windows 7 operating system with Intel Core i5-2400 CPU, 4 GB RAM, on a desktop computer. Both the nonlinear MPC and the proposed piecewise MPC provide comparable dynamic performance. Their computational times are summarized in Table 6. From Table 6, it can be seen that the computational time of each proposed MPC step is less than the nonlinear MPC on all control horizon lengths. It is more obvious when the horizon length is extended. The comparative results present the computation efficiency of the proposed method. To obtain the average computational time of each MPC step, the repeated numbers of MPC steps are 1200 .

5.3.3. Superiority. In this subsection, we verify the superiority of the proposed method through comparison with the classical PID method.

The velocity difference between each locomotive and the reference velocity value is plotted in the following figures.

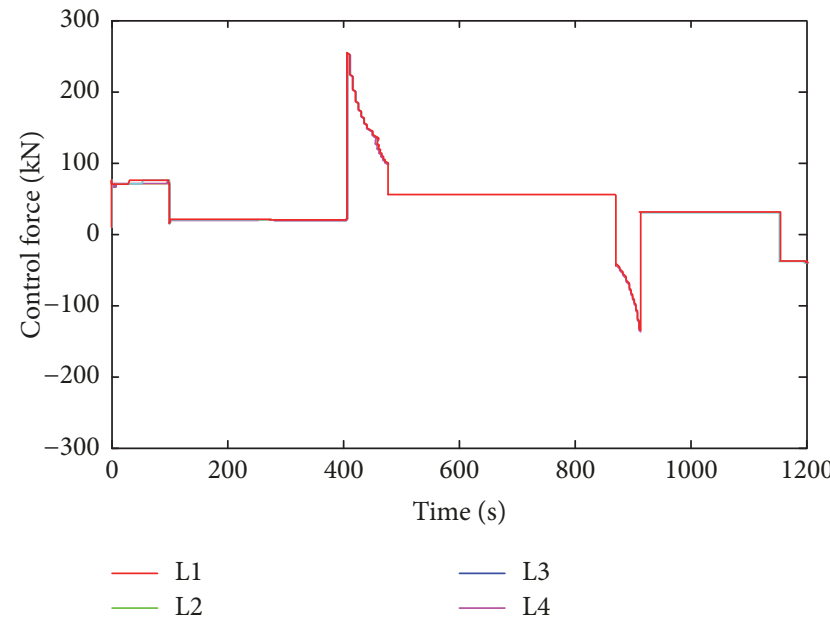

FIGURE 12: The force output of each locomotive with power limit.

Figures 13(a) and 13(b) show the simulation results under PID and the proposed MPC methods, respectively. From Figure 13(a), the velocities of four locomotives have severe chattering at time $400 \mathrm{~s}$ and $900 \mathrm{~s}$ when the reference velocity varies, which can further cause drastic changes of the coupler and endanger safe operation of high-speed trains. The proposed method in this paper presents good control performance as shown in Figure 13(b). From this figure, we can see that the velocities of four locomotives do not change frequently and can converge to the desired velocity quickly. This control performance is conducive to the safe operation of the train.

5.3.4. Stochastic Perturbations. In this subsection, we consider the performance of the proposed method with external stochastic perturbations. The stochastic perturbations are added in the system dynamics (6). The maximum amplitude of the perturbation is assumed to be 1 . Considering the constant term $\beta_{j}=0.0044$, the perturbation is large enough to evaluate the robustness of the proposed method. The external stochastic perturbations are produced by using the Matlab function randn $(n)$, which can create an $n$-by- $n$ matrix of normally distributed random numbers with mean equal to zero and standard deviation equal to 1 . The simulation result of the system with the stochastic perturbations is shown in Figure 14. From this figure, we can see that the practical velocities of locomotives can track the reference values well, though the stochastic perturbations exist. The simulation results show the good robustness of the proposed method. 


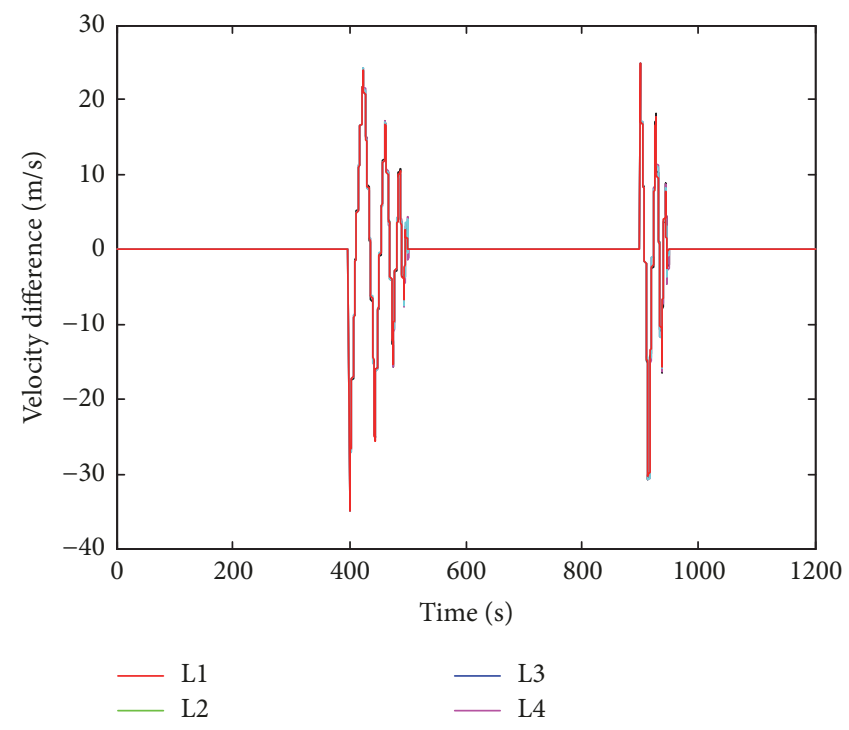

(a) PID method

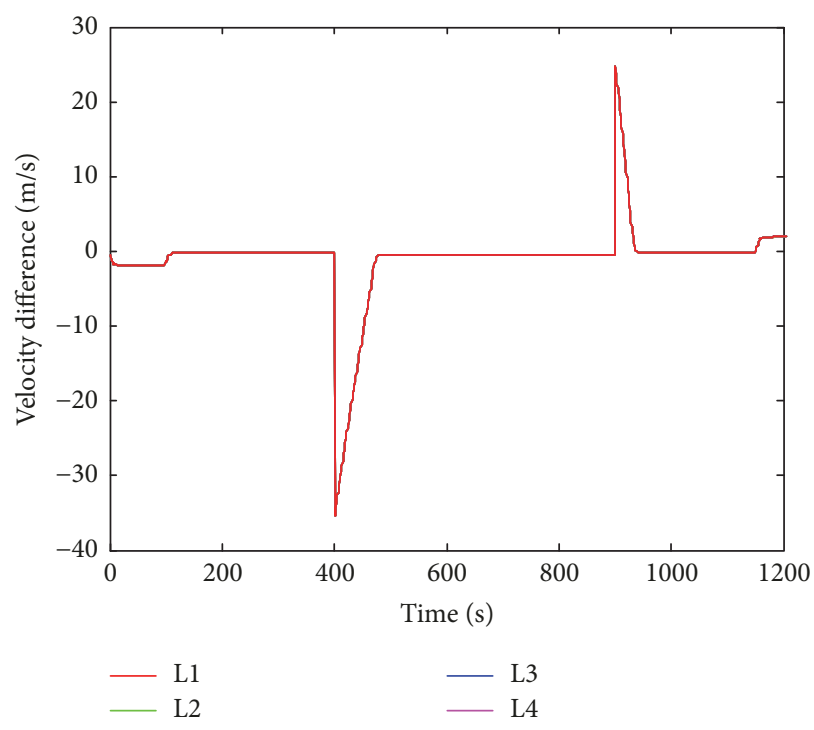

(b) MPC method

FIGURE 13: Comparison of MPC method with PID method in velocity error.

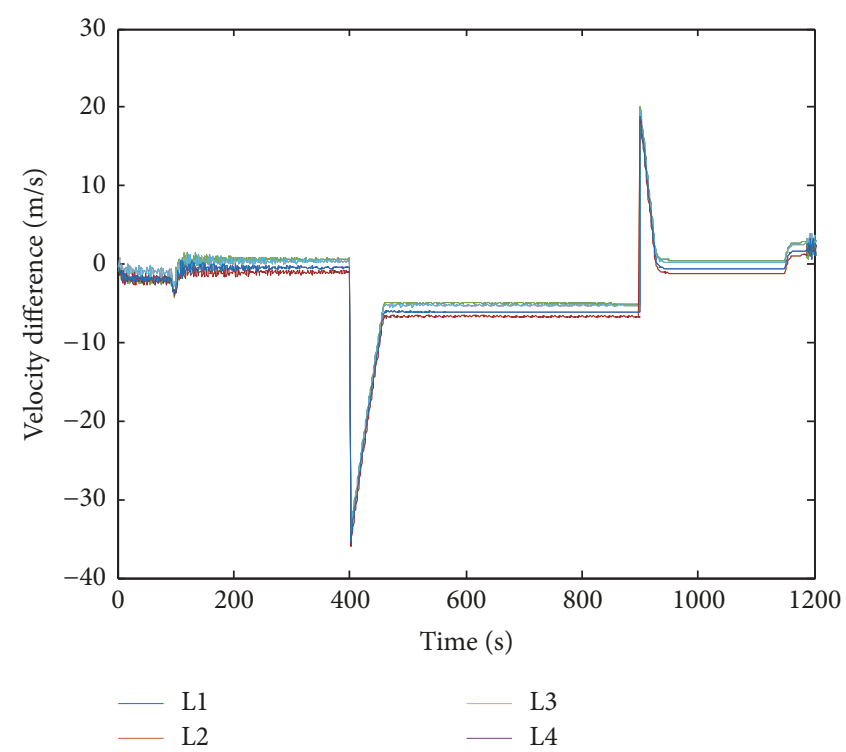

FIGURE 14: The velocity of each vehicle under MPC method with external stochastic perturbations.

\section{Conclusion}

In this paper, we investigated the automatic cruising control problem of high-speed trains with the aim of tracking the reference speed and minimizing the energy consumption and guaranteeing the safe operation of high-speed trains. First, we built the piecewise linearization of train resistance and developed the piecewise multiple-point model of trains. Due to the multiple constraints and performance metrics, we proposed a model predictive control method to design the ATO controller. In order to improve the accuracy of the method, we introduced the binary variable and transformed the piecewise model into a mixed logical dynamical model and guarantee that the submodel in the prediction process is still switchable. Then we analyze the operation objective and constraints and transformed the tracking control problem into a constrained finite-time optimal control problem. The optimization problem is solved by the integer linear planning method, and the optimal control solution is obtained. Simulations in the Matlab verified the effectiveness of the proposed algorithm.

In this work, we just consider the nonlinear characteristics of resistance when constructing the train model and neglect the control delay. In the future work, more realistic train models will be investigated to incorporate more nonlinear characteristics and the controller robustness will be improved under time delay. 


\section{Disclosure}

Part of this paper [2] was presented at the 29th Chinese Control and Decision Conference, Chongqing, China, May 2017.

\section{Conflicts of Interest}

The authors declare that there are no conflicts of interest regarding the publication of this paper.

\section{Acknowledgments}

This work is partially supported by the National Natural Science Foundation of China (Grants nos. 61379111, 61672537, and 61672539).

\section{References}

[1] W. Song, J. Ma, L. Zhou, and X. Feng, "Deadbeat Predictive Power Control of Single-Phase Three-Level Neutral-PointClamped Converters Using Space-Vector Modulation for Electric Railway Traction," IEEE Transactions on Power Electronics, vol. 31, no. 1, pp. 721-732, 2016.

[2] Z. Xu, Z. Huang, K. Gao, S. Li, R. Zhang, and W. Liu, "Optimal operation of high-speed train using hybrid model predictive control," in Proceedings of the 2017 29th Chinese Control And Decision Conference (CCDC), pp. 3642-3647, Chongqing, China, May 2017.

[3] Y. Luo and H. Xu, "Direct robust adaptive control of high speed train based on nonlinear and time-varying models," International Journal of Control and Automation, vol. 6, no. 4, pp. 397-410, 2013.

[4] W. Zhao and C. Gao, "A New Control Method of Automatic Train Operation in Urban Rail Transit Based on Improved Generalized Predictive Control Theory," in Proceedings of the 2013 International Conference on Electrical and Information Technologies for Rail Transportation (EITRT2013)-Volume I, vol. 287 of Lecture Notes in Electrical Engineering, pp. 567-573, Springer Berlin Heidelberg, Berlin, Heidelberg, 2014.

[5] Y. Wang, R. Luo, Z. Yu, and B. Ning, "Study on ato control algorithm with consideration of atp speed limits," Journal of the China Railway Society, vol. 5, p. 017, 2012.

[6] A. Albrecht, P. Howlett, P. Pudney, X. Vu, and P. Zhou, "The key principles of optimal train control-Part 1: Formulation of the model, strategies of optimal type, evolutionary lines, location of optimal switching points," Transportation Research Part B: Methodological, vol. 94, pp. 482-508, 2016.

[7] C.-D. Yang and Y.-P. Sun, "Robust cruise control of high speed train with hardening/softening nonlinear couple," in Proceedings of the American Control Conference, vol. 1999, pp. 2200-2204.

[8] C. Yang and $\mathrm{Y}$. Sun, "Mixed $\mathrm{H}_{2} / \mathrm{H}_{\infty}$ cruise controller design for high speed train," International Journal of Control, vol. 74, no. 9, pp. 905-920, 2001.

[9] C-J. Lin, S-H. Tsai, C-L. Chen, and T.-H. S. Li, "Extended sliding-mode controller for high speed train," in Proceedings of the 2010 International Conference on System Science and Engineering (ICSSE), pp. 475-480, Taipei, Taiwan, July 2010.

[10] Q. Song, Y.-D. Song, T. Tang, and B. Ning, "Computationally inexpensive tracking control of high-speed trains with traction/braking saturation," IEEE Transactions on Intelligent Transportation Systems, vol. 12, no. 4, pp. 1116-1125, 2011.

[11] X. Zhuan and X. Xia, "Optimal scheduling and control of heavy haul trains equipped with electronically controlled pneumatic braking systems," IEEE Transactions on Control Systems Technology, vol. 15, no. 6, pp. 1159-1166, 2007.

[12] X. Zhuan and X. Xia, "Cruise control scheduling of heavy haul trains," IEEE Transactions on Control Systems Technology, vol. 14, no. 4, pp. 757-766, 2006.

[13] M. Chou and X. Xia, "Optimal cruise control of heavy-haul trains equipped with electronically controlled pneumatic brake systems," Control Engineering Practice, vol. 15, no. 5, pp. 511-519, 2007.

[14] X. Zhuan and X. Xia, "Speed regulation with measured output feedback in the control of heavy haul trains," Automatica, vol. 44, no. 1, pp. 242-247, 2008.

[15] G. M. Scheepmaker, R. M. Goverde, and L. . Kroon, "Review of energy-efficient train control and timetabling," European Journal of Operational Research, vol. 257, no. 2, pp. 355-376, 2017.

[16] M. Faieghi, A. Jalali, S. K. Mousavi Mashhadi, and D. Baleanu, "Passivity-based cruise control of high speed trains," Journal of Vibration and Control, vol. 24, no. 3, pp. 492-504, 2018.

[17] M. Faieghi, A. Jalali, and S. K. E. D. M. Mashhadi, "Robust adaptive cruise control of high speed trains," ISA Transactions, vol. 53, no. 2, pp. 533-541, 2014.

[18] T. Tao and H. Xu, "Adaptive fault-tolerant cruise control for a class of high-speed trains with unknown actuator failure and control input saturation," Mathematical Problems in Engineering, vol. 2014, Article ID 481315, 2014.

[19] L. Fan, "Iterative learning and adaptive fault-tolerant control with application to high-speed trains under unknown speed delays and control input saturations," IET Control Theory \& Applications, vol. 8, no. 9, pp. 675-687, 2014.

[20] S. Li, L. Yang, Z. Gao, and K. Li, “Optimal Guaranteed Cost Cruise Control for High-Speed Train Movement," IEEE Transactions on Intelligent Transportation Systems, vol. 17, no. 10, pp. 2879-2887, 2016.

[21] H. Ye and R. Liu, "Nonlinear programming methods based on closed-form expressions for optimal train control," Transportation Research Part C: Emerging Technologies, vol. 82, pp. 102-123, 2017.

[22] Y. Zhao, T. Wang, and H. R. Karimi, "Distributed cruise control of high-speed trains," Journal of The Franklin Institute, vol. 354, no. 14, pp. 6044-6061, 2017.

[23] L. J. Zhang and X. T. Zhuan, "Optimal operation of heavyhaul trains equipped with electronically controlled pneumatic brake systems using model predictive control methodology," IEEE Transactions on Control Systems Technology, vol. 22, no. 1, pp. 13-22, 2014.

[24] S. Li, B. De Schutter, L. Yang, and Z. Gao, "Robust Model Predictive Control for Train Regulation in Underground Railway Transportation," IEEE Transactions on Control Systems Technology, vol. 24, no. 3, pp. 1075-1083, 2016.

[25] A. Bemporad, F. Borrelli, and M. Morari, "Model predictive control based on linear programming the explicit solution," Institute of Electrical and Electronics Engineers Transactions on Automatic Control, vol. 47, no. 12, pp. 1974-1985, 2002.

[26] E. D. Sontag, "Nonlinear regulation: the piecewise linear approach," Institute of Electrical and Electronics Engineers Transactions on Automatic Control, vol. 26, no. 2, pp. 346-358, 1981. 
[27] C. E. García, D. M. Prett, and M. Morari, "Model predictive control: theory and practice-a survey," Automatica, vol. 25, no. 3, pp. 335-348, 1989.

[28] A. Bemporad and M. Morari, "Control of systems integrating logic, dynamics, and constraints," Automatica, vol. 35, no. 3, pp. 407-427, 1999.

[29] X.-g. Li, D.-j. Gao, and Y.-h. Wang, "Predictive control for hybrid system based on mixed logic dynamic model," Control and Decision, vol. 17, no. 3, pp. 315-319, 2002.

[30] Y. Wang, B. De Schutter, T. J. J. van den Boom, and B. Ning, "Optimal trajectory planning for trains - A pseudospectral method and a mixed integer linear programming approach," Transportation Research Part C: Emerging Technologies, vol. 29, pp. 97-114, 2013.

[31] F. D. Torrisi and A. Bemporad, "HYSDEL—a tool for generating computational hybrid models for analysis and synthesis problems," IEEE Transactions on Control Systems Technology, vol. 12, no. 2, pp. 235-249, 2004.

[32] A. Bemporad, Hybrid Toolbox-Usern's Guide, 2003, Hybrid toolbox-userns guide.

[33] L. Li, W. Dong, Y. Ji, Z. Zhang, and L. Tong, "Minimal-energy driving strategy for high-speed electric train with hybrid system model," IEEE Transactions on Intelligent Transportation Systems, vol. 14, no. 4, pp. 1642-1653, 2013. 


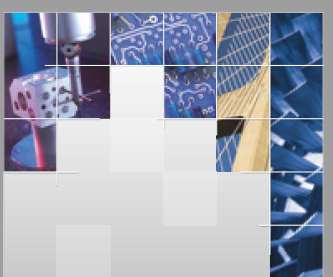

\section{Enfincering}
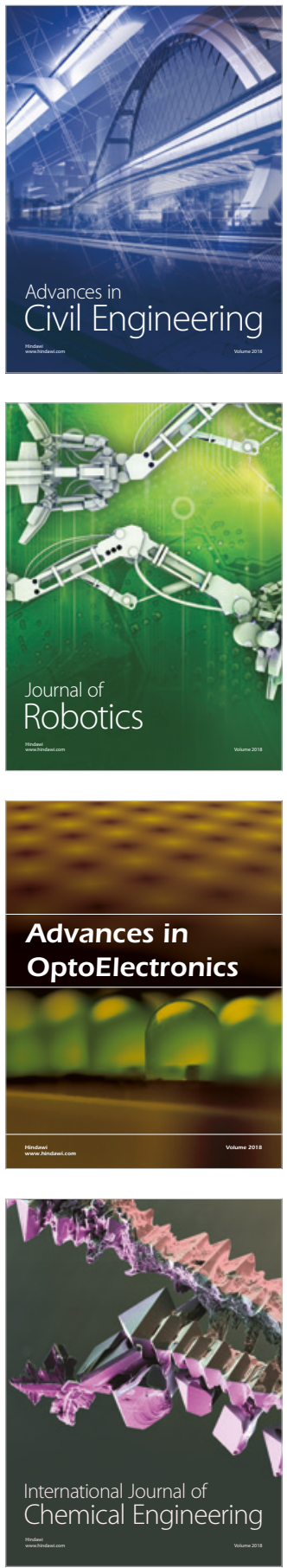

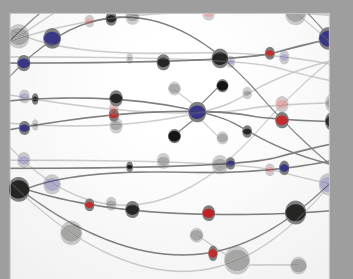

\section{Rotating \\ Machinery}

The Scientific World Journal

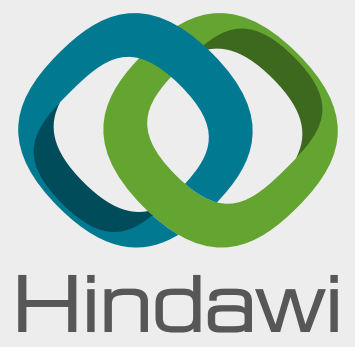

Submit your manuscripts at

www.hindawi.com
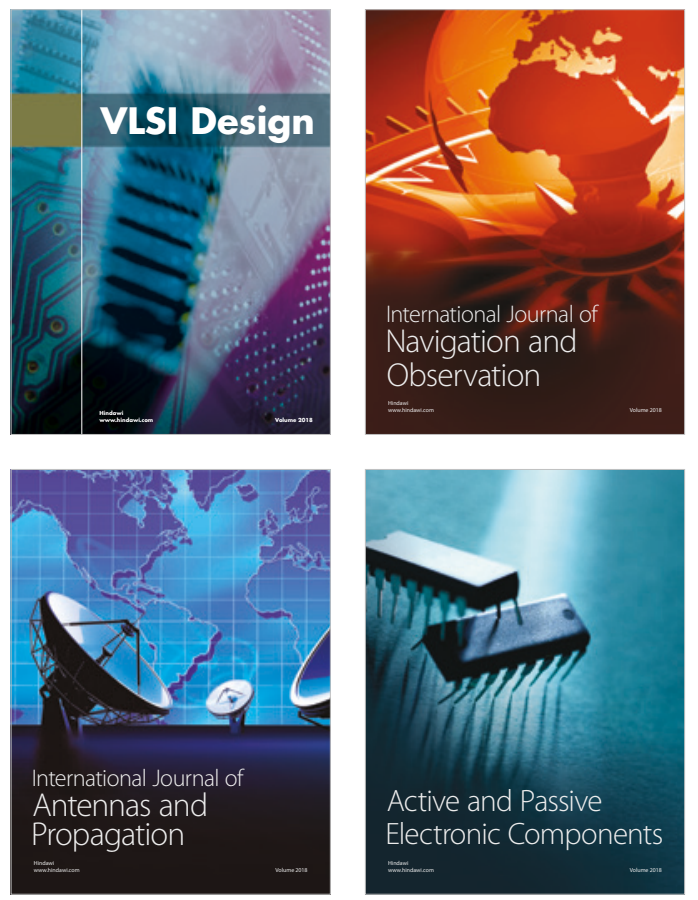
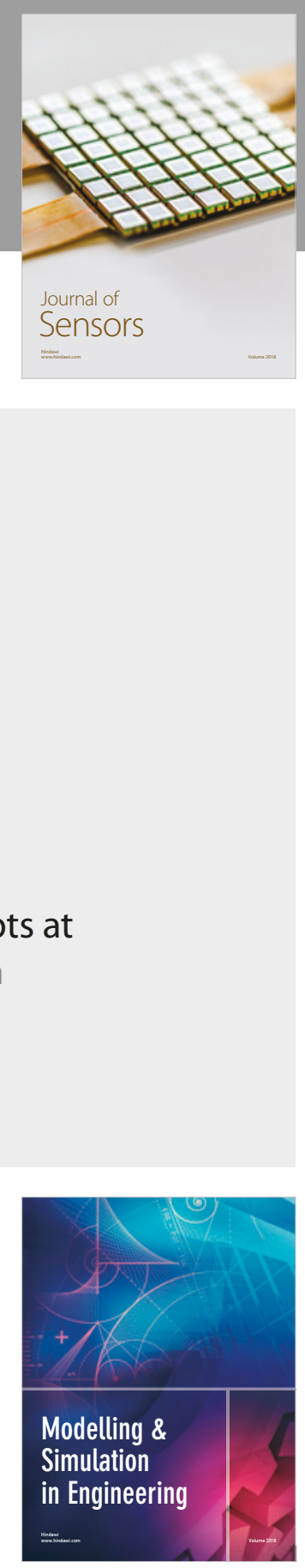

\section{Advances \\ Multimedia}
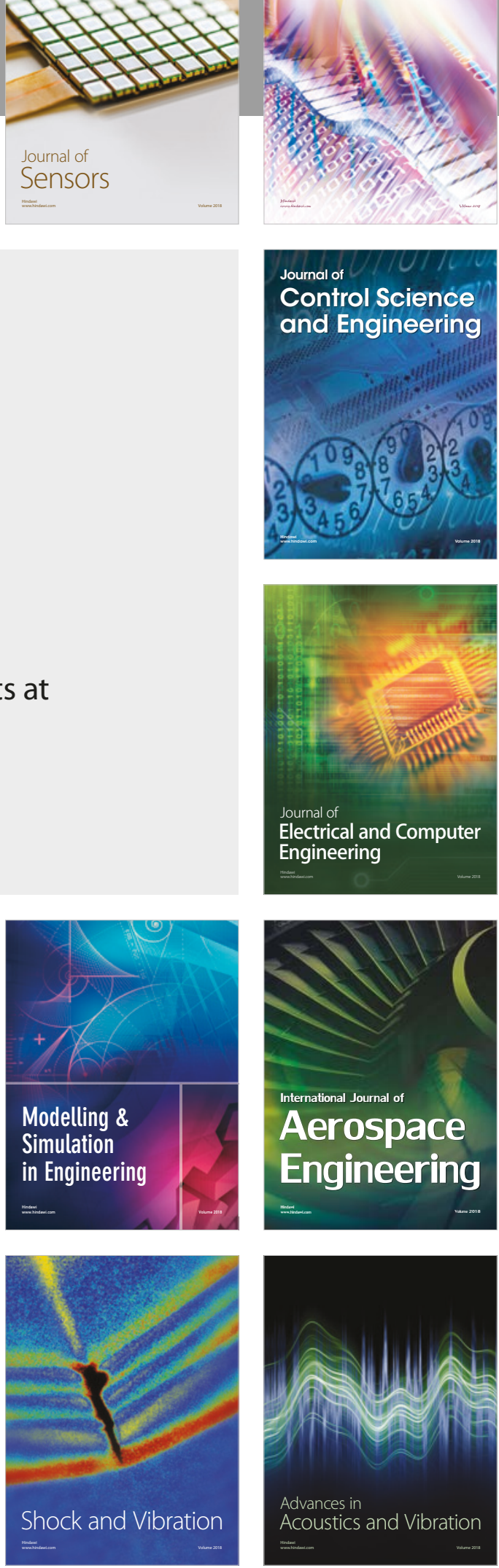